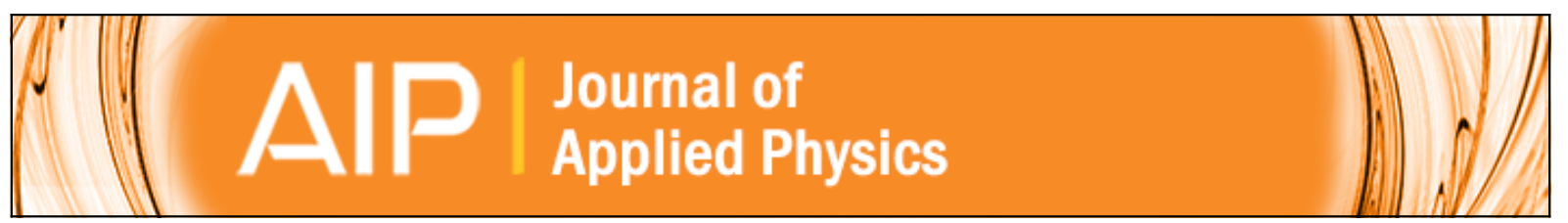

Magnetic and elastic anisotropy in magnetorheological elastomers using nickel-based nanoparticles and nanochains

Romina A. Landa, Paula Soledad Antonel, Mariano M. Ruiz, Oscar E. Perez, Alejandro Butera, Guillermo Jorge, Cristiano L. P. Oliveira, and R. Martín Negri

Citation: Journal of Applied Physics 114, 213912 (2013); doi: 10.1063/1.4839735

View online: http://dx.doi.org/10.1063/1.4839735

View Table of Contents: http://scitation.aip.org/content/aip/journal/jap/114/21 ?ver=pdfcov

Published by the AIP Publishing

AlP 


\title{
Magnetic and elastic anisotropy in magnetorheological elastomers using nickel-based nanoparticles and nanochains
}

\author{
Romina A. Landa, ${ }^{1}$ Paula Soledad Antonel, ${ }^{1}$ Mariano M. Ruiz, ${ }^{1}$ Oscar E. Perez, ${ }^{2}$ \\ Alejandro Butera, ${ }^{3}$ Guillermo Jorge,${ }^{4}$ Cristiano L. P. Oliveira, ${ }^{5}$ and R. Martín Negri ${ }^{1, a)}$ \\ ${ }^{1}$ Departamento de Química Inorgánica, Analítica y Química Física, Facultad de Ciencias Exactas y \\ Naturales, Instituto de Quimica Física de Materiales, Ambiente y Energía (INQUIMAE), Universidad de \\ Buenos Aires, Ciudad Universitaria, Pabellón II, C1428EGA Buenos Aires, Argentina \\ ${ }^{2}$ Departamento de Industrias, Facultad de Ciencias Exactas y Naturales, Universidad de Buenos Aires, \\ Buenos Aires, Argentina \\ ${ }^{3}$ Centro Atómico Bariloche (Comisión Nacional de Energía Atómica. Argentina) and Instituto Balseiro, \\ Universidad Nacional de Cuyo, Mendoza, Argentina \\ ${ }_{4}^{4}$ Instituto de Ciencias, Universidad Nacional de General Sarmiento, Buenos Aires, Argentina \\ ${ }^{5}$ Grupo de Fluidos Complexos, Instituto de Física, Universidade de São Paulo, São Paulo, Brazil
}

(Received 25 September 2013; accepted 17 November 2013; published online 6 December 2013)

\begin{abstract}
Nickel (Ni) based nanoparticles and nanochains were incorporated as fillers in polydimethylsiloxane (PDMS) elastomers and then these mixtures were thermally cured in the presence of a uniform magnetic field. In this way, macroscopically structured-anisotropic PDMS$\mathrm{Ni}$ based magnetorheological composites were obtained with the formation of pseudo-chains-like structures (referred as needles) oriented in the direction of the applied magnetic field when curing. Nanoparticles were synthesized at room temperature, under air ambient atmosphere (open air, atmospheric pressure) and then calcined at $400{ }^{\circ} \mathrm{C}$ (in air atmosphere also). The size distribution was obtained by fitting Small Angle X-ray Scattering (SAXS) experiments with a polydisperse hard spheres model and a Schulz-Zimm distribution, obtaining a size distribution centered at $(10.0 \pm 0.6) \mathrm{nm}$ with polydispersivity given by $\sigma=(8.0 \pm 0.2) \mathrm{nm}$. The SAXS, X-ray powder diffraction, and Transmission Electron Microscope (TEM) experiments are consistent with single crystal nanoparticles of spherical shape (average particle diameter obtained by TEM: $(12 \pm 1) \mathrm{nm}$ ). Nickel-based nanochains (average diameter: $360 \mathrm{~nm}$; average length: $3 \mu \mathrm{m}$, obtained by Scanning Electron Microscopy; aspect ratio $=$ length/diameter $\sim 10$ ) were obtained at $85^{\circ} \mathrm{C}$ and ambient atmosphere (open air, atmospheric pressure). The magnetic properties of Ni-based nanoparticles and nanochains at room temperature are compared and discussed in terms of surface and size effects. Both Ni-based nanoparticles and nanochains were used as fillers for obtaining the PDMS structured magnetorheological composites, observing the presence of oriented needles. Magnetization curves, ferromagnetic resonance (FMR) spectra, and strain-stress curves of low filler's loading composites ( $2 \% \mathrm{w} / \mathrm{w}$ of fillers) were determined as functions of the relative orientation with respect to the needles. The results indicate that even at low loadings it is possible to obtain magnetorheological composites with anisotropic properties, with larger anisotropy when using nanochains. For instance, the magnetic remanence, the FMR field, and the elastic response to compression are higher when measured parallel to the needles (about 30\% with nanochains as fillers). Analogously, the elastic response is also anisotropic, with larger anisotropy when using nanochains as fillers. Therefore, all experiments performed confirm the high potential of nickel nanochains to induce anisotropic effects in magnetorheological materials. ( 2013 AIP Publishing LLC. [http://dx.doi.org/10.1063/1.4839735]
\end{abstract}

\section{INTRODUCTION}

Magnetorheological elastomers are composite materials formed by dispersions of magnetic micro or nano compounds (fillers) in an organic viscoelastic non-fluid matrix. ${ }^{1-21}$ These materials display anisotropy in their magnetic, electric, or mechanical properties, usually achieved by applying external fields during the formation of the composite. While the use of nickel $(\mathrm{Ni})$ nanocompounds is continuously receiving

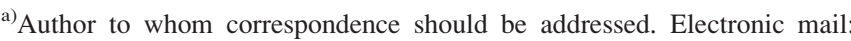
rmn@qi.fcen.uba.ar. Tel.: 54-11-4576-3358. FAX: 54-11-4576-3341
}

attention for different applications; ${ }^{22-26}$ their use as fillers in magnetorheological composites has not been fully explored yet. Kchit and Bossis ${ }^{1}$ used Ni microparticles covered with silver (10 microns), but the use of Ni nanostructures, such as nanoparticles (NPs), nanochains (NC), or nanowires as fillers in magnetorheological elastomers has not been yet reported in the literature. One possible reason is that compared with the chemical synthesis of nanostructures of noble metals or transition metal oxides, obtaining nickel nanostructures with high purity and low size dispersion presents some difficulties. Usually, nickel (II) compounds are treated with a reducing agent in the presence of other compounds and under 
specific protocols which include heating, argon atmosphere, $\mathrm{pH}$ control, mixing of solvents, microwave heating, selfassembly processes, and electrodeposition. ${ }^{27-37}$

In the present work, we have synthesized Ni nanoparticles and nanochains using synthesis protocols under ambient air atmosphere, which were initially explored by Sidhaye et $a l .{ }^{38}$ for Ni nanoparticles and Wang et al. ${ }^{29}$ for nanochains. The obtained materials were then used as fillers for the fabrication of magnetorheological composites by dispersion of the Ni nanostructures into an elastomer polymer (polydimethylsiloxane, PDMS) followed by thermal curing in the presence of a uniform magnetic field. In this way, it was possible to obtain magnetic elastomers with anisotropic elastic and magnetic properties provided by the formation of pseudo-chains of the fillers aligned in the direction of the magnetic field applied during curing. These magnetorheological elastomers must be differentiated from ferrofluids and other extensively studied viscoelastic materials (like magnetohydrodynamic gels), since magnetorheological elastomers are non-fluid at room temperature.

Thus, the aims of the present work are first to present the synthesis and characterization of $\mathrm{Ni}$ nanoparticles and nanochains; second, to demonstrate that magnetic and elastic anisotropic effects can be induced in magnetorheological elastomers using low concentrations of $\mathrm{Ni}$ nanostructures as fillers, comparing the effects of using $\mathrm{Ni}$ nanoparticles and $\mathrm{Ni}$ nanochains. These nanostructures are grouped when curing the matrix in the presence of a magnetic field, forming pseudo-chains. These pseudo-chains are referred as "needles" from now on, to avoid confusion with the term "nanochains" (reserved for one of the fillers used). A scheme illustrating the needles formed by nanoparticles or nanochains is shown in Figure 1.

\section{EXPERIMENTAL SECTION}

Nickel nitrate hexahydrate $\left(\mathrm{Ni}\left(\mathrm{NO}_{3}\right)_{2} \cdot 6 \mathrm{H}_{2} \mathrm{O}\right)$, sodium dodecyl sulfate (SDS), oleic oil, sodium borohydride $\left(\mathrm{NaBH}_{4}\right)$, poly-vinylpyrrolidone (PVP), hydrazine monohydrate $98 \%\left(\mathrm{~N}_{2} \mathrm{H}_{4} \cdot \mathrm{H}_{2} \mathrm{O}\right)$, sodium hydroxide, and ethylene glycol were purchased from Sigma Aldrich (analytical grade).

\section{A. Synthesis of Ni nanoparticles}

Nickel compounds were synthesized following Sidhaye et al $^{38}$ with some modifications (e.g. larger reactants concentrations). The ratio $\mathrm{Ni}(\mathrm{II})$ :SDS was maintained fixed (1:10) in all the synthesis. Typically, an aqueous mixture of $5 \times 10^{-3} \mathrm{M} \mathrm{Ni(II),} 5 \times 10^{-2} \mathrm{M}$ SDS and $5 \times 10^{-4} \mathrm{M}$ oleic oil was vigorous stirred at the temperature of synthesis, $\mathbf{T}_{\text {synth }}=25^{\circ} \mathrm{C}$. In all synthesis, $\mathrm{NaBH}_{4}$ (reducing agent) was added and the color of the solution turned black immediately after its addition. After $1 \mathrm{~h}$ under stirring, the solutions were subjected to centrifugation at $10000 \mathrm{G}$, the pellets washed with MilliQ water and re-centrifuged several times under the same conditions. The inorganic material was dried in a vacuum oven at room temperature and heated afterwards at $400{ }^{\circ} \mathrm{C}$ for $5 \mathrm{~h}$ under atmospheric air. The apparition in the final material of boron (B) compounds, like Ni-B phases or borates, was not observed by $\mathrm{X}$-ray powder diffraction (XRD); boron was neither detected by Energy Dispersive Spectroscopy (EDS; detector: Oxford Instrument, model INCAx-Sight; detection limit: $0.1 \% w / w)$. Both XRD and Small Angle X-ray Scattering (SAXS) experiments show diffractograms coincident with those expected for nickel, without observing peaks or shoulders that could be associated to nickel oxides or hydroxides.

\section{B. Synthesis of Ni nanochains}

Nickel nanochains were synthesized based on the protocol reported by Wang et al. ${ }^{29}$ In our case, $0.25 \mathrm{~g}$ of PVP and $0.363 \mathrm{~g}$ of $\mathrm{Ni}\left(\mathrm{NO}_{3}\right)_{2} \cdot 6 \mathrm{H}_{2} \mathrm{O}$ were dispersed under vigorous stirring in $22.5 \mathrm{ml}$ of ethylene glycol at $85^{\circ} \mathrm{C}$ (temperature of synthesis). After $15 \mathrm{~min}, 0.3 \mathrm{ml}$ of distilled water and $1.3 \mathrm{ml}$ of $\mathrm{N}_{2} \mathrm{H}_{4} \cdot \mathrm{H}_{2} \mathrm{O}(98 \%)$ were added. At this point, the color of the solution turned light violet due to the formation of $\mathrm{Ni}(\mathrm{II})-\mathrm{N}_{2} \mathrm{H}_{4}$ complexes. ${ }^{39}$ Finally, after $10 \mathrm{~min}, 0.4 \mathrm{~g}$ of $\mathrm{NaOH}$ were added, and the $\mathrm{Ni}(\mathrm{II})-\mathrm{N}_{2} \mathrm{H}_{4}$ complexes react with the $\mathrm{OH}^{-}$, resulting in the reduction of the complexes to metallic $\mathrm{Ni}^{40}$ The reaction mixture was kept under vigorous stirring during $2 \mathrm{~h}$ at the temperature of synthesis. Then, the solid material was separated by centrifugation at $10000 \mathrm{G}$, the first 4 cycles washing with water, and the last one using

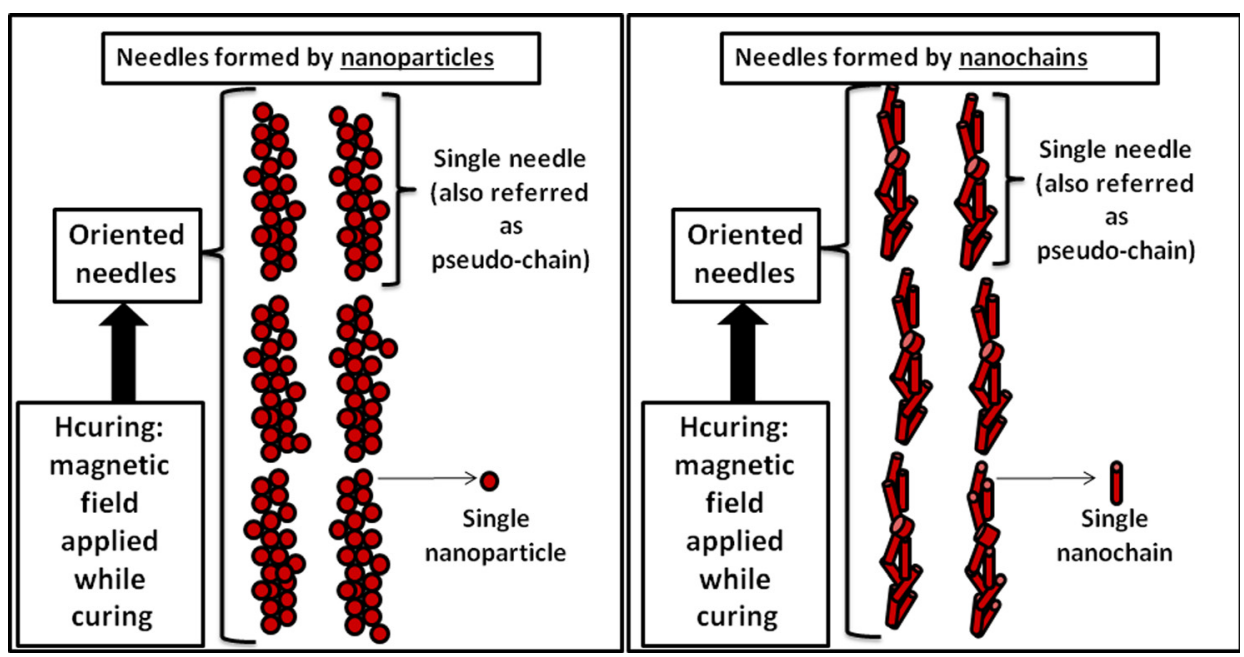

FIG. 1. Scheme of the pseudo-chains (referred as needles in the manuscript) formed after curing PDMS composites in the presence of a magnetic field ( $\mathrm{H}_{\text {curing }}$ ) when using: (a) Ni nanoparticles (left); (b) Ni nanochains (right), as fillers. 
ethanol. The black solid was finally dried using a vacuum oven at room temperature for $24 \mathrm{~h}$. The formation of nanochains results from adding PVP. As in the case of the nanoparticles, no presence of oxides or hydroxides was detected by XRD.

\section{Preparation of PDMS-Ni composites}

PDMS base and cross linker agent (Sylgar 184, Dow Corning) were mixed in proportions of $1: 10(w / w)$ at room temperature and then loaded with $2 \% \mathrm{w} / \mathrm{w}$ of the fillers: $\mathrm{Ni}$ nanoparticles or Ni nanochains. Air bubbles were eliminated for every preparation before curing by placing the PDMSfiller mixture in a vacuum oven at room temperature until complete removal of bubbles. Additional care concerning the possible presence of air bubbles inside the composite was taken during loading the mold for curing and checked after curing by observing multiple longitudinal and transversal cuts of the cured samples using Scanning Electron Microscopy (SEM) and optical microscopy. Bubbles were not detected in any of the prepared composites.

The device designed for curing the composite in presence of a uniform magnetic field was described previously, ${ }^{2-5}$ hence a short description is presented here. In all cases, the fluid mixture PDMS-fillers was placed inside a cylindrical mould (dimensions: $1 \times 3 \mathrm{~cm}$ ) which is part of a designed device that allows curing the samples at a given temperature in the presence of an applied magnetic field while rotating the sample at constant speed (approximately $30 \mathrm{rpm}$ ) for homogenizing. The samples were cured at $(75 \pm 5)^{\circ} \mathrm{C}$ during $4 \mathrm{~h}$ in the presence of a uniform magnetic field $\left(\mathbf{H}_{\text {curing }}=0.35 \mathrm{~T}\right)$. The magnetic field was generated with a standard Varian low impedance electromagnet (model V3703) provided with a set of pole pieces with a diameter of $10 \mathrm{~cm}$. These kinds of electromagnets are known to provide highly homogeneous steady magnetic fields. The magnetic field magnitude between the pole pieces was measured by a Hall-probe electronic gaussmeter. A composite with $2 \% \mathrm{w} / \mathrm{w}$ of $\mathrm{Ni}$ nanoparticles was cured without applying a magnetic field for comparison.

\section{XRD, transmission electron microscope (TEM), and SEM}

XRD analysis of the nanoparticles was performed with a Philips X-Pert diffractometer using $\mathrm{CuK}_{\alpha}$ radiation $(\lambda=0.154056 \mathrm{~nm})$ and the average size of the crystallites determined by the Scherrer equation. A Philips EM 301 TEM was used to examine the morphology and also the size distribution of the nanoparticles and nanochains. The size distribution was determined in each case by counting on at least 200 particles. The morphology of Ni nanochains and of the PDMS-Ni composites was studied using a Field Emission SEM (FESEM; Zeiss Supra 40 Gemini).

\section{E. SAXS experiments and modeling}

SAXS experiments were performed in a laboratory based equipment, NANOSTAR ${ }^{\mathrm{TM}}$ from Bruker at the Laboratory of Crystallography, Institute of Physics, University of São Paulo.
The powder samples of Ni nanoparticles (the powder was not diluted) were exposed to the X-Rays using a Scotch Tape ${ }^{\mathrm{TM}}$ to support the samples. The background was taken using the scattering from the tape alone. The data were recorded using exposition times of $900 \mathrm{~s}$. The data treatment was performed using the package SUPERSAXS (developed by Oliveira \& Pedersen, University of Sao Paulo). The experimental data are displayed as Intensity, $I(q)$, versus the momentum transfer $q, q=(4 \pi / \lambda) \sin \theta$, where $\lambda$ is the radiation wavelength and $2 \theta$ is the scattering angle. The measured $q$ interval was $0.085-3.4 \mathrm{~nm}^{-1}$. A model based on a polydispersed system of hard spheres (PHS) was used to describe the experimental data, using the Schulz-Zimm size distribution and the model developed by Percus-Yevick (PY). ${ }^{41-43}$ Fitting of experimental data by these models allows obtaining the average sphere radius $\boldsymbol{R}_{\boldsymbol{a}}$, the polydispersivity $\boldsymbol{\sigma}_{\boldsymbol{R}}$, and the effective hard sphere volume fraction $\boldsymbol{\eta}$. Details and model equations used for analyzing the data of SAXS experiments are presented in supplementary material. ${ }^{53}$

\section{F. Magnetic properties of nanostructures and composites}

A LakeShore 7400 Vibrating Sample Magnetometer (VSM) was used for recording magnetization curves at room temperature. In both cases, Ni nanoparticles and nanochains, a weighted mass of powder samples (5-20 mg) was packed with Teflon tape and mounted in the VSM sample-holder. On the other hand, in the case of Ni-PDMS composites, a slide of the sample was placed in the VSM sample-holder and the magnetization measured at different angles in order to investigate magnetic anisotropy effects. In all cases, the magnetization curves were taken from positive saturation at $1 \mathrm{~T}$, in steps of $25 \mathrm{mT}$ or less, with an integration constant of $10 \mathrm{~s}$ for each applied magnetic field.

Ferromagnetic-resonance (FMR) spectra at $24.1 \mathrm{GHz}$ of the NI-PDMS composites were recorded with a Bruker ESP300 spectrometer at room temperature. The samples were cut in slabs of $2 \mathrm{~mm} \times 2 \mathrm{~mm}$ and a thickness of a fraction of a millimeter, maintaining the needles (formed during curing) within or perpendicular to the surface of the slabs. The samples were placed at the center of the resonant cavity. The slab plane could be either parallel or perpendicular to the microwave excitation, according to the desired angular variation. Angular variations with respect to the external DC magnetic field were made around the slab normal or within the slab plane.

\section{G. Texture analysis}

Composites samples for texture analysis were prepared by cutting the cured composites using a specially designed holder in order to obtain pieces of similar thickness $(\cong 2 \mathrm{~mm})$ and similar areas $\left(0.78 \mathrm{~cm}^{2}\right.$ and $\left.0.83 \mathrm{~cm}^{2}\right)$ for the perpendicular and parallel cuts with respect to the direction of the applied magnetic field during curing, respectively.

The texture analysis was performed using a Stable Microsystems TA-XT2i Texture Analyzer which compresses the sample at a constant compression speed $(100 \mu \mathrm{m} / \mathrm{s})$ in the range between $8 \%$ and $40 \%$ of the initial thickness. ${ }^{44}$ 
Different compression-decompression cycles were performed at least in duplicate to characterize the material recovery, possible ruptures, and elastic hysteresis.

\section{RESULTS AND DISCUSSION}

In the first sections, the results concerning the synthesis and properties of the fillers (Ni nanoparticles and nanochains) are presented (Secs. III A and III B). The results obtained for PDMS-Ni magnetorheological elastomers are described and discussed in the last sections (Secs. III C-III E).

\section{A. Chemical and morphological characterization of $\mathrm{Ni}$ nanoparticles and nanochains}

The XRD patterns (Figure 2(a)) indicate that the Ni nanoparticles are essentially crystalline single phases with XRD peaks corresponding to the face centered cubic (fcc) structure of Ni (ICDD 01-1260).

Concerning the average crystallite size of the Ni phase, peaks that correspond to $\mathrm{Ni}$ fcc are well distinguished. The mean crystallite size was estimated using the Scherrer equation from the major diffraction peak of the base, (111) at $44.7^{\circ}$, obtaining $(13 \pm 1) \mathrm{nm}$, in close agreement with the average particle size obtained by TEM (Figures 3(a) and 3(b)) and SAXS (Figure 2(b)). The crystallite size of the synthesized Ni nanoparticles is smaller than those obtained by Sidhaye et al $^{38}$ which used the same Ni(II):SDS ratio but different reactant concentrations.

Figure 3(a) shows TEM images of Ni-based nanoparticles prepared at room temperature. The size distribution present a maximum at $(12 \pm 1) \mathrm{nm}$, a value coincident with the average crystallite size calculated by Scherrer, $(13 \pm 1)$ $\mathrm{nm}$ as indicated above, suggesting that the Ni-based nanoparticles are single-crystal domains. This is confirmed by the SAXS analysis (Figure 2(b)). The experimental $I_{\text {exp }}(\mathrm{q})$ data were fitted by using a PHS model, with a Schulz-Zimm size distribution and a structure factor given by the PY, described in supplementary material as mentioned in Sec. IIE. Thus, the resulting model for fitting the SAXS data, referred as PHS-PY model, considers that the only relevant interaction is between the Ni-based particles taken as hard spheres without substructures. This model provided the best fitting of the scattering data. Other attempts were tried by using models that consider fractal structures (particles aggregates forming structures referred as fractals), obtaining unsatisfactory fittings. This indicates that the Ni-based nanoparticles can be considered as hard-spheres, without presenting aggregations with ramified structures. In other words, the fact that excellent (and the best) fits were obtained by modeling the $\mathrm{Ni}$ based nanoparticles by the PHS-PY model means that the system is formed by polydisperse particles of spherical shape.

The recovered values of $\boldsymbol{R}_{\boldsymbol{a} v}$ and $\boldsymbol{\sigma}_{\boldsymbol{R}}$ for Ni nanoparticles using PHS-PY are $\boldsymbol{R}_{\boldsymbol{a} v}=(5.0 \pm 0.3) \mathrm{nm}$ and $\boldsymbol{\sigma}_{\boldsymbol{R}}=(4.0 \pm 0.1)$ $\mathrm{nm}$. Thus, the SAXS modeling indicates a reasonable polydispersivity in sizes, rendering a polydispersivity of $\sim 56 \%$ $\left(\mathbf{z}=\left(\mathbf{R}_{\mathbf{a v}} / \boldsymbol{\sigma}_{\mathbf{R}}\right)^{2}-1=0.56\right)$. The average hard-sphere size $2 \boldsymbol{R}_{\boldsymbol{a} v}=(10.0 \pm 0.6) \mathrm{nm}$ is in excellent agreement with the position of the maximum for the size distribution obtained by TEM $((12 \pm 1) \mathrm{nm})$ and also with the average crystallite size calculated from Scherrer equation $((13 \pm 1) \mathrm{nm})$, confirming that the nanoparticles can be considered as monocrystalline domains of approximately spherical size. It is worth to note that the values obtained by SAXS have better precision than those obtained from TEM since in SAXS the information comes from a huge number of particles which provides the data that are analyzed in the reciprocal space. The agreement between the particle sizes obtained by SAXS, XRD, and TEM confirms that the particles possesses a well defined crystalline structure, that is, particles presents very good crystal quality.

A low value of the effective volume fraction occupied by the particles, $\boldsymbol{\eta}$, was recovered from SAXS, $(\boldsymbol{\eta}=0.105$ $\pm 0.003)$, which can be attributed to sample preparation effects (empty space between nanoparticles) and/or to surface roughness effects in each nanoparticle.

The TEM and SEM images of Ni nanochains are presented in Figures 3(b) and 3(c). The SEM image with a high magnification $(200000 \times)$ (Figure $3(\mathrm{c})$ ) allows seeing the morphology, noting the presence of interacting layers that form the whole nanochain. Two hundred nanochains were considered for determining its average diameter $(360 \mathrm{~nm})$ and length $(3 \mu \mathrm{m})$. EDS of the nanochains indicated a very low oxygen atomic percentage (less than $5 \%$ ).
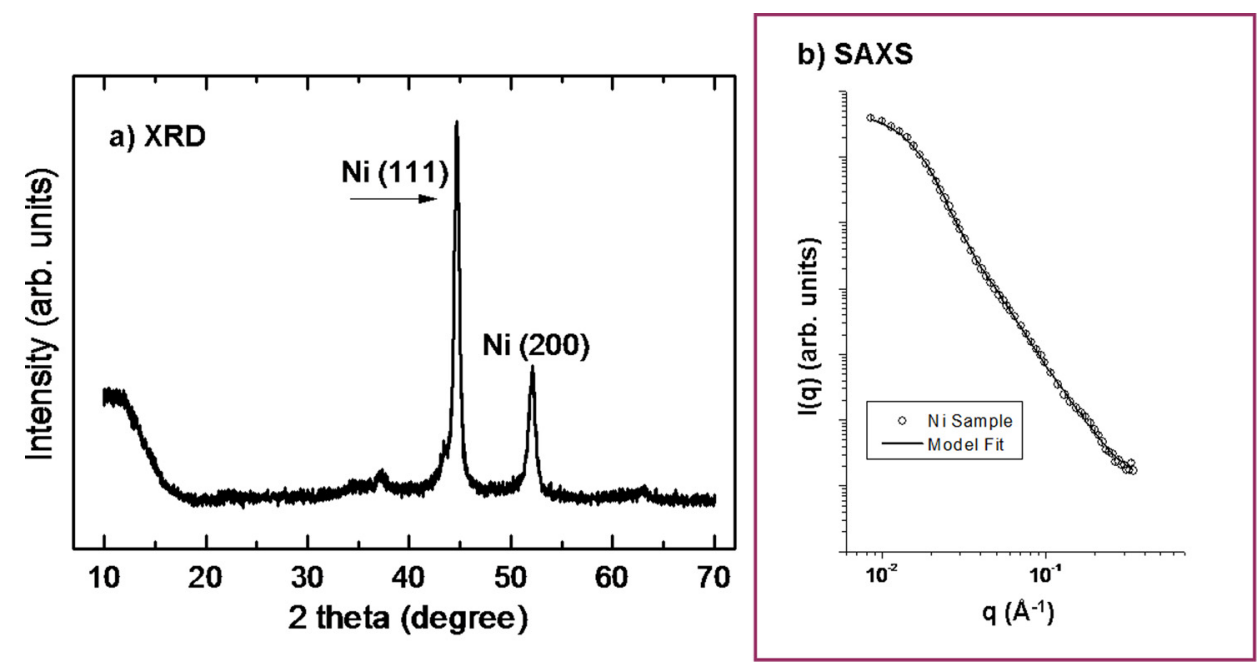

FIG. 2. (a) XRD pattern of the Nibased nanoparticles synthesized at $25^{\circ} \mathrm{C}$. (b) SAXS intensity, $I(q)$ vs. the momentum transfer $q, q=(4 \pi / \lambda) \sin \theta$ (where $\lambda$ is the radiation wavelength and $2 \theta$ is the scattering angle) for the $\mathrm{Ni}$ nanoparticles. 

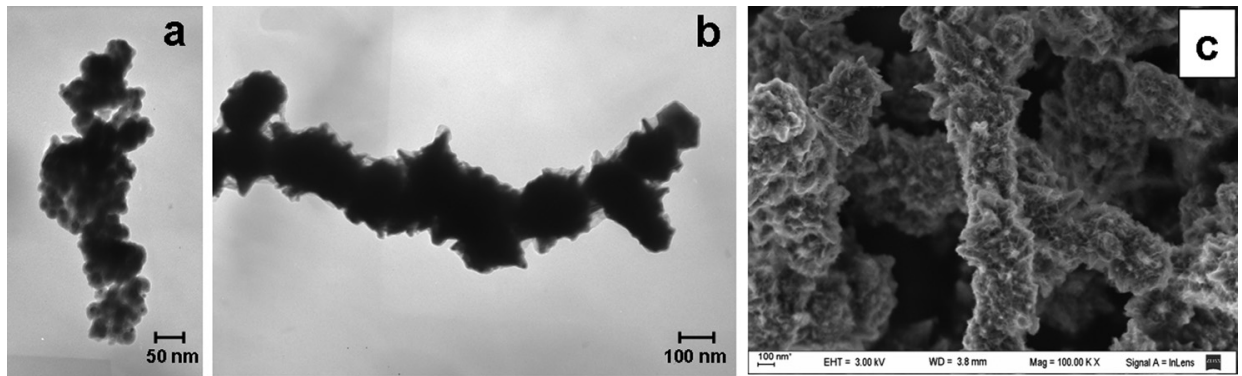

FIG. 3. (a) TEM images of Ni nanoparticles. (b) and (c) SEM images of Ni nanochains.

\section{B. Magnetic characterization of $\mathrm{Ni}$ nanoparticles and nanochains.}

The magnetization loops at room temperature for the Ni-based nanoparticles and Ni-based nanochains are presented in Figures 4(a) and 4(b), respectively. Magnetic hysteresis loops were observed indicating the ferromagnetic behavior of Ni-based nanoparticles and nanochains at room temperature. Table I allows comparing the saturation magnetization, $\mathbf{M}_{\mathbf{s}}$, remanent magnetization, $\mathbf{M}_{\mathbf{r}}$, the ratio $\left(\mathbf{M}_{\mathbf{r}} / \mathbf{M}_{\mathbf{s}}\right)$, and coercive field, $\mathbf{H}_{\mathbf{c}}$, for Ni-based nanoparticles, Ni-based nanochains, and Ni-bulk.

$\mathbf{M}_{\mathbf{s}}$ decreases from bulk to nanochains to nanoparticles. In particular, the value of $\mathbf{M}_{\mathbf{s}}$ for the nanoparticles $(20 \mathrm{emu} / \mathrm{g})$ is lower than half of the value for bulk and nanochains (about $50 \mathrm{emu} / \mathrm{g}$ ). This decrease of $\mathbf{M}_{\mathbf{s}}$ with the average size of the material has been observed in the cases of $\mathrm{Ni}$ and $\mathrm{CoFe}_{2} \mathrm{O}_{4}$ nanoparticles. ${ }^{4}{ }^{414} \mathrm{An}$ explanation given by $\mathrm{Lu}$ et $a l .{ }^{46}$ proposes a possible partial suppression of the magnetization in one or several surface layers of the nanomaterial due to a spin disordered structure in its surface. Since the surface/volume ratio increases significantly from bulk (microparticles) to nanoparticles then the effect of a non-magnetic or superparamagnetic superficial layer is expected to be larger in the nanoparticles with the consequent relative diminishing of $\mathbf{M}_{\mathrm{s}}$ as observed in Table I.

A crude estimation of the number of non-ferromagnetic layers can be made by assuming cubic NP of volume $\mathbf{V}=10 \times 10 \times 10 \mathrm{~nm}^{3}$, cubic unit cells with parameter $\boldsymbol{a}_{\mathrm{uc}}=3 \AA \quad(\mathrm{Ni}-\mathrm{fcc})$, and the ratio $\mathbf{M}_{\mathrm{s}}(\mathbf{N P}) / \mathbf{M}_{\mathbf{s}}(\mathbf{b u l k})$ $=\left(\mathbf{V}-\mathbf{n}\left(\boldsymbol{a}_{\mathbf{u c}}\right)^{3}\right) / \mathbf{V}$ with $\mathbf{n}$ the number of non-ferromagnetic layers. These assumptions render about $7 \times 10^{3}$ unit cells at the total surface, $\mathbf{n}=2 \times 10^{4}$ (with $\mathbf{M}_{\mathbf{s}}(\mathbf{N P}) / \mathbf{M}_{\mathbf{s}}(\mathbf{b u l k})=20 / 50$ $=0.4)$ and therefore a number of about $3 \quad(\cong 2 / 0.7)$ non-ferromagnetic layers is estimated in the nanoparticles.
On the other hand, $\mathbf{H}_{\mathbf{c}}$ increases from bulk to nanochains to nanoparticles. This trend can be explained assuming that the nanoparticles constitute magnetic monodomains (although with ferromagnetic behavior at room temperature) and considering that the reversal magnetization mechanism in ferromagnetic systems is different for large multi-domain materials (bulk and nanochains) than for monodomain ones (nanoparticles). For the multi-domain systems, the main mechanism of magnetization reversal is the movement of domain walls, while for the monodomain ferromagnetic particles the mechanism is the coherent rotation of the spins, a process which requires higher coercive fields than domain walls motion. This qualitative description is in agreement with the tendencies for $\mathbf{H}_{\mathbf{c}}$ observed in Table I.

The relative influence of those factors in $\mathbf{H}_{\mathbf{c}}$, are hard to introduce quantitatively because of the difficulties for evaluating reasonably the values of microscopic parameters such as the effective magnetic anisotropy constant, $\mathbf{K}_{\text {eff }}$, which may differ from the bulk value due to surface effects as those described before $\left(\mathbf{K}_{\text {bulk }}=4.510^{3} \mathrm{erg} / \mathrm{cm}^{3}\right.$ for fcc-Ni, averaged in all directions ${ }^{47}$ ). For example, the maxima contribution of the crystal magnetic anisotropy in magnetic single domains is expected to be (0.7-0.5) $\left(2 \mathbf{K}_{\text {bulk }} / \mathbf{M}_{\mathbf{s}}\right)$, expressing $\mathbf{M}_{\mathrm{s}}$ in $\mathrm{emu} / \mathrm{cm}^{3}$ (a Ni density of $8.9 \mathrm{~g} / \mathrm{cm}^{3}$ was used for converting the units of $\mathbf{M}_{\mathrm{s}}$ of Table I to $\mathrm{emu} / \mathrm{cm}^{3}$ ). For the nanochains, we measured $\mathbf{H}_{\mathbf{c}}=150 \mathrm{Oe}$ which is higher than the respective value of $(0.7-0.5)\left(2 \mathbf{K} / \mathbf{M}_{\mathbf{s}}\right)(=144-103 \mathrm{Oe})$ indicating that another contribution, besides the magnetic crystalline anisotropy, should be considered and suggesting contribution from shape anisotropy to $\mathbf{H}_{\mathbf{c}}$ in the nanochains. On the other hand, for the case of nanoparticles is $\mathbf{H}_{\mathbf{c}}=180$ Oe while $(0.7-0.5)\left(2 \mathbf{K} / \mathbf{M}_{\mathbf{s}}\right)=354-253$ Oe, thus, $\mathbf{H}_{\mathbf{c}}$ for the nanoparticle is in agreement with the prediction of a single-domain with a contribution of magnetic crystalline
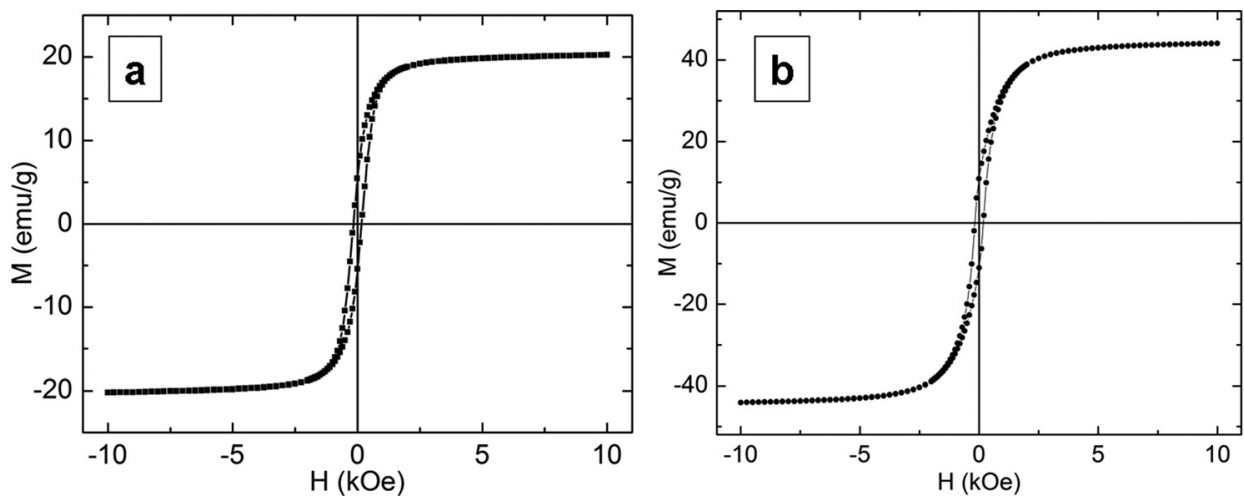

FIG. 4. Magnetization curves at $25^{\circ} \mathrm{C}$. (a) Ni nanoparticles. (b) Ni nanochains. 
TABLE I. Magnetic parameters at $298 \mathrm{~K}$.

\begin{tabular}{|c|c|c|c|}
\hline & $\mathrm{M}_{\mathrm{s}}^{\mathrm{a}}(\mathrm{emu} / \mathrm{g})$ & $\mathrm{M}_{\mathrm{r}} / \mathrm{M}_{\mathrm{s}}^{\mathrm{b}}$ & $\mathrm{H}_{\mathrm{c}}{ }^{\mathrm{c}}(\mathrm{Oe})$ \\
\hline Ni bulk ${ }^{\mathrm{d}}$ & 55 & 0.05 & 100 \\
\hline $\mathrm{Ni} \mathrm{NC}$ & 49 & 0.16 & 150 \\
\hline Ni NP & 20 & 0.27 & 180 \\
\hline $2 \% w / w$ of NC-PDMS composites ( $\mathrm{H} \perp$ to the needles) & $\ldots$ & 0.22 & 150 \\
\hline $2 \% w / w$ NC-PDMS composites (H // to the needles) & $\ldots$ & 0.38 & 150 \\
\hline $2 \% w / w$ NP-PDMS composites ( $\mathrm{H} \perp$ to the needles) & $\ldots$ & 0.29 & 180 \\
\hline $2 \% w / w$ NP-PDMS composites (H // to the needles) & $\ldots$ & 0.39 & 180 \\
\hline
\end{tabular}

${ }^{\mathrm{a}}$ Saturation magnetization, $\mathrm{M}_{\mathrm{s}}$

${ }^{b}$ Remanent magnetization relative to the saturation magnetization, $M_{r} / M_{s}$.

${ }^{\mathrm{c}}$ Coercive field, $\mathrm{H}_{\mathrm{c}}$

${ }^{\mathrm{d}}$ From Ref. 45.

anisotropy to $\mathbf{H}_{\mathbf{c}}$ that in principle cannot be neglected. This is also in agreement with the spherical (non anisotropic) shape of the nanoparticles obtained by SAXS. Summarizing, the present analysis strongly suggest morphology magnetic anisotropy in the nanochains while magnetic crystalline anisotropy for the nanoparticles as the main contributions to magnetic anisotropy in the respective cases.

All the experimental results presented in this section are consistent with nanoparticles that constitute magnetic monodomains, ${ }^{48}$ whereas the nanochains consist of multidomains. The observed tendencies, larger $\mathbf{H}_{\mathbf{c}}$ and $\mathbf{M}_{\mathbf{r}} / \mathbf{M}_{\mathbf{s}}$ and the lower $\mathbf{M}_{\mathbf{s}}$ for the nanoparticles in comparison with the nanochains, are qualitatively interpreted within that context, suggesting magnetic morphology anisotropy has a relevant influence in the magnetic properties of the nanochains, while those of the nanoparticles are mainly influenced by surface effects.

\section{Morphology of anisotropic PDMS-Ni composites}

Macroscopic pseudo-chains (needles) of inorganic filler material were only obtained when applying a magnetic field during curing, while composites with uniform distribution of fillers (without macroscopic aggregation) were obtained when curing in absence of the magnetic field. Optical photographs and SEM images of the structured composites using $\mathrm{Ni}$ nanoparticles as fillers are shown in Figures 5 and 6, respectively.

The needles are oriented along the direction of the magnetic field and can be observed by the naked eye; top and

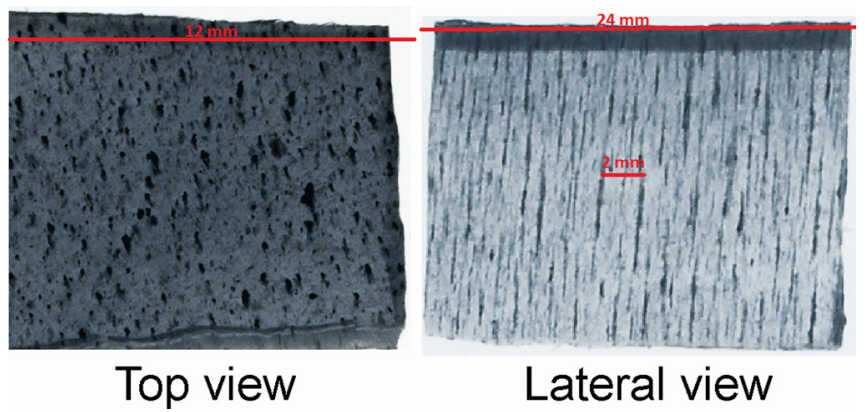

FIG. 5. Photographs of the structured composites, observing the magnetically aligned needles of $\mathrm{Ni}$ nanoparticles in PDMS (2\% w/w of nanoparticles). (a) Top view, perpendicular to the needles. (b) Lateral view, parallel to the needles. lateral views of the composites are shown in Figure 5, recorded with a digital photographic camera. It is worth to remark that non-uniform dispersion of the magnetic needles or domains formation was never observed for matrixes cured in the presence of the magnetic field. The density of needles was calculated in several different portions of the samples, obtaining always similar values independent of the chosen portion.

All the fillers appear incorporated into the needles (confirmed by SEM, Figure 6). SEM images show that the needles are formed by conglomerates of the individual fillers (nanoparticles or nanochains).

Figures 6(a) and 6(b) show examples of top and lateral views of the needles, respectively, for composites loaded with $2 \% w / w$ of Ni-nanoparticles. These images allow performing some dimensional estimation. For instance, the calculated number of needles that cross a surface perpendicular to its orientation is about $(5 \pm 1)$ needles $/ \mathrm{mm}^{2}$ and they are separated by an average distance of $(1.0 \pm 0.3) \mathrm{mm}$. The average length of the needles is about $1.5-2.0 \mathrm{~mm}$ with average diameter of $25 \mu \mathrm{m}$. Using these averaged dimensions, a volume fraction $\left(\phi_{\mathrm{v}}\right)$ of about $0.1 \%-0.5 \%$ is estimated for the case of $2 \% w / w$ PDMS-Ni-based nanoparticles composites (similar volume fractions were estimated for the case of Ni-based nanochains as fillers). The obtained values of $\phi_{\mathbf{v}}\left(\phi_{\mathbf{v}} \approx 0.003\right.$ $=0.3 \%$ for $2 \% w / w$ of filler; $\boldsymbol{W}=0.02=$ filler mass fraction) are in agreement with a crude estimation assuming the density of the needles equal to the density of bulk $\mathrm{Ni}$ $\left(\delta_{\mathrm{Ni}}=8.9 \mathrm{~g} / \mathrm{cm}^{3}\right)$ and considering that the density of the composite is $\boldsymbol{\delta}_{\text {composite }} \approx 1.2 \mathrm{~g} / \mathrm{cm}^{3}$ calculated by weighing and measuring the geometrical dimensions of the composites. Under these assumptions it can be estimated: $\boldsymbol{\phi}_{\mathbf{v}}=\boldsymbol{W}$ $\left(\delta_{\text {composite }} / \delta_{\mathrm{Ni}}\right)=0.02 \times(1.2 / 8.9)=0.0027$, which is in excellent agreement with the value indicated above.

Figure 6(c) shows the interface between a needle and PDMS, observing a clear separation (non-adhesion) between the polymer matrix and the inorganic needles. This is highly relevant in connection with the absence of elastic hysteresis, as discussed in Sec. III E. Figure 6(d) illustrates that the needles formed by the nanochains cross through the elastomer's top surface (which is perpendicular to the field applied during curing). 

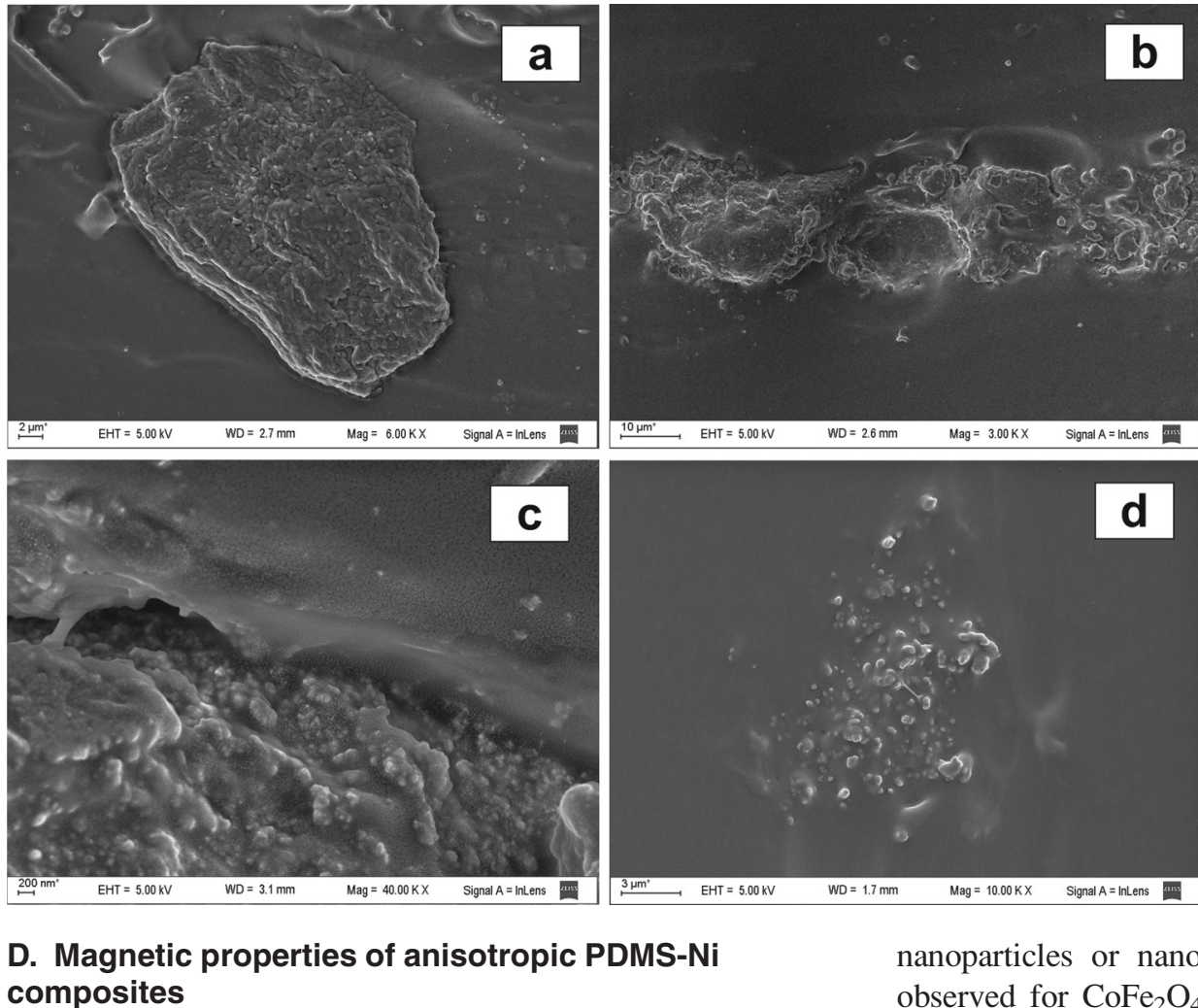

\section{Magnetic properties of anisotropic PDMS-Ni composites}

The magnetic properties of the composites were measured as function of the angle $(\alpha)$ between the needle's alignment and the sensing magnetic field by VSM and FMR (Figures 7 and 8, respectively, with sensing field parallel to the needles, $\alpha=0$ ). The composites present ferromagnetic behavior with magnetic anisotropy since variation with $\alpha$ was observed for the magnetization curves, $\mathbf{M}(\mathbf{H}), \mathbf{M}_{\mathrm{s}}, \mathbf{M}_{\mathbf{r}}, \mathbf{M}_{\mathbf{r}} / \mathbf{M}_{\mathbf{s}}$ and the resonance field in FMR, $\mathbf{H}_{\mathbf{r}}$ (whatever using

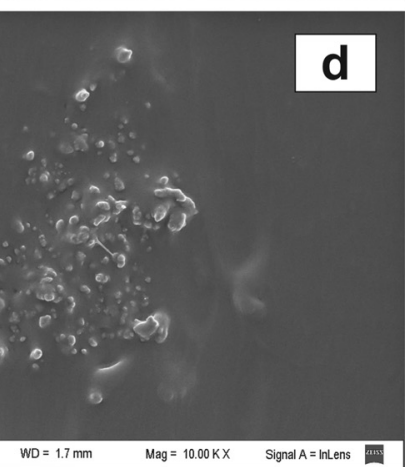

FIG. 6. SEM images of the structured Ni-PDMS composites. (a) Needle formed by Ni nanoparticles (top view), dimensions: $23.6 \times 33.9 \mu \mathrm{m}$. (b) idem (a) but in a lateral view. (c) Image showing the interface between a needle formed by $\mathrm{Ni}$ nanoparticles and the PDMS matrix. (d) Top view from a SEM image of a Ni nanochains-PDMS composite, showing that the needles (formed by nanochains) cross through the top surface of the composite.
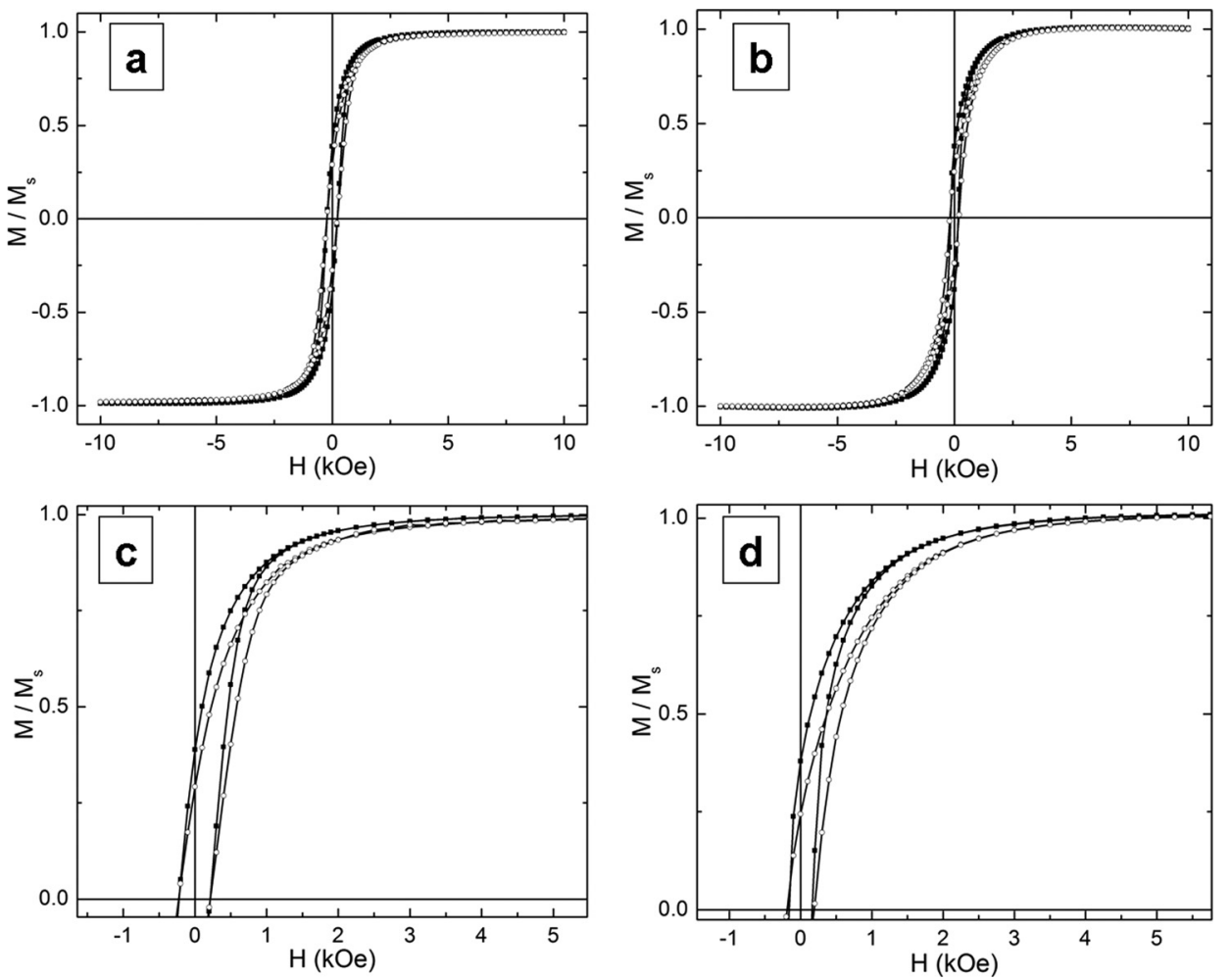

nanoparticles or nanochains as fillers). This behavior was observed for $\mathrm{CoFe}_{2} \mathrm{O}_{4}$ nanoparticles in $\mathrm{PDMS}^{4}$ and $\mathrm{Ni}$ in a similar matrix. ${ }^{14}$ The only parameter that remains isotropic and with the same value for the composites and the filler's powder is the coercive field, $\mathbf{H}_{\mathbf{c}}$, in agreement with the picture described in Sec. III C of needles formed by conglomerates of the fillers.

On the other hand, $\mathbf{M}_{\mathbf{s}}, \mathbf{M}_{\mathbf{r}}$, and $\mathbf{M}_{\mathbf{r}} / \mathbf{M}_{\mathbf{s}}$ are larger when measured at $\alpha=0^{\circ}$ than at other angles, with minimum values at $\alpha=90^{\circ}$, observing higher anisotropy when using

FIG. 7. Normalized magnetization curves $\left(\mathrm{M} / \mathrm{M}_{\mathrm{s}}\right.$ vs $\left.\mathrm{H}\right)$ of the composites for two different angles, $\alpha$, between the applied magnetic field $(\mathrm{H})$ and the direction of the needles $\left(\alpha=0^{\circ}\right.$ : ———; $\alpha=90^{\circ}:-\bigcirc-$ ). (a) and (c) corresponds to Ni-based nanoparticles as fillers, while Ni-based nanochains were used in (b) and (d). Figs. 6(c) and 6(d) are magnifications of Figs. 6(a) and 6(b), respectively. 

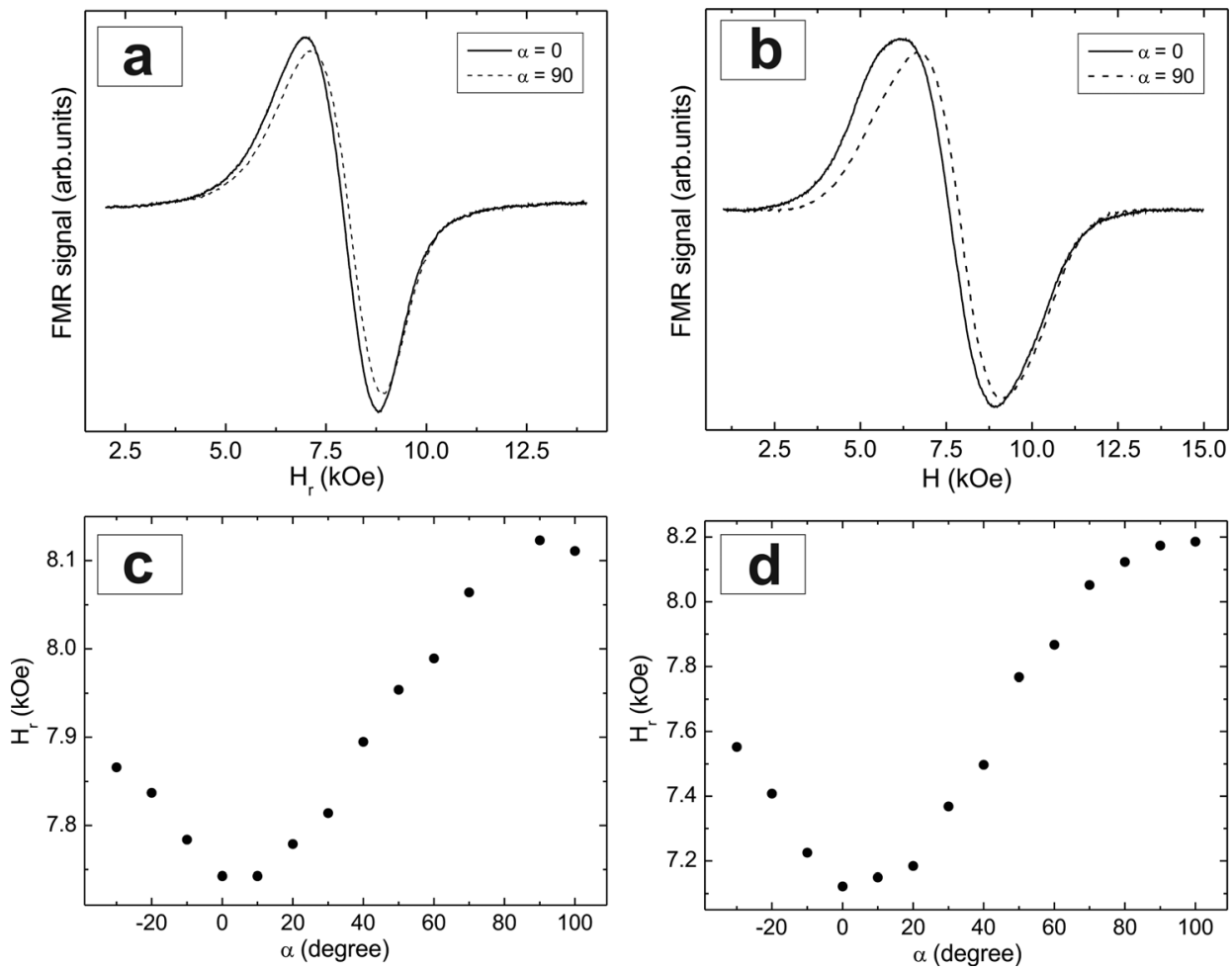

FIG. 8. (a) and (b) FMR signals $(24.1 \mathrm{GHz})$ for PDMS structured composites using Ni-based nanoparticles and Ni-based nanochains as fillers, respectively (the angle $\alpha$ is defined as in Figure 6). (c) and (d) Resonance field $\left(\mathrm{H}_{\mathrm{r}}\right)$ as function of $\alpha$ for composites with Ni-based nanoparticles and nanochains, respectively. nanochains than nanoparticles as fillers. For example, the remanence ratio $\left(\mathbf{M}_{\mathbf{r}} / \mathbf{M}_{\mathbf{s}}\right)$ is a $37 \%$ lower for $\alpha=90^{\circ}$ than for $\alpha=0^{\circ}$ in the composites loaded with $\mathrm{Ni}$ nanochains and a $26 \%$ lower when loading with Ni nanoparticles (these values correspond to $2 \% \mathrm{w} / \mathrm{w}$ loading in both cases). Another illustration of the anisotropic magnetic behavior is the difference in the resonance field, $\mathbf{H}_{\mathbf{r}}$, between $\alpha=0^{\circ}$ and $\alpha=90^{\circ}$ measured by FMR at $24.1 \mathrm{GHz}$. For this particular frequency the differences are $12 \%$ and $5 \%$ for nanochains and nanoparticles as fillers, respectively (Figures 8(a) and 8(b)).

The shift in the FMR spectra when varying $\alpha$ is given by the change of the resonance field, $\mathbf{H}_{\mathbf{r}}$, with $\alpha$ (Figures $8(\mathrm{c})$ and $8(\mathrm{~d})$ ). From these figures, it is possible to estimate the effective anisotropy field for both fillers using the expression $\boldsymbol{H}_{\text {eff }}=2 / 3\left[\mathbf{H}_{\mathbf{r}}\left(\alpha=90^{\circ}\right)-\mathbf{H}_{\mathbf{r}}\left(\alpha=0^{\circ}\right)\right]{ }^{49,50} \mathrm{We}$ obtained $\boldsymbol{H}_{\boldsymbol{e f f}} \sim 600 \mathrm{Oe}$ and $\boldsymbol{H}_{\boldsymbol{e f f}} \sim 260 \mathrm{Oe}$ for the nanochains and the nanoparticles in PDMS composites, respectively indicating that the nanochains form needles with a considerably larger anisotropy. Both anisotropy values are still smaller than those expected for a perfectly homogeneous nickel cylinder, which theoretically should have shape anisotropy fields $\mathbf{H}_{\mathbf{s}}=2 \pi \mathbf{M}_{\mathbf{s}} \sim 3070 \mathrm{Oe}$ and $1260 \mathrm{Oe}$ (assuming $\mathbf{M}_{\mathbf{s}}=488 \mathrm{emu} / \mathrm{cm}^{3}$ and $200 \mathrm{emu} / \mathrm{cm}^{3}$ ) for nanochains and nanoparticles. Note, however, that the ratio between the two effective anisotropy fields, $(600 / 260=2.3)$ is almost coincident with the ratio between the saturation magnetizations $(488 / 200=2.4$, see Table I and Sec. III B) suggesting that the differences in the anisotropy come mostly from the reduction of $\mathbf{M}_{\mathbf{s}}$ in the nanoparticles relative to the nanochains and not from a change in the shape of the needles. The considerably increase in the line width, $\Delta \mathrm{H}$, for the magnetoelastomers with nanochains in comparison with those using nanoparticles $(\Delta H=2600$ and
1500 Oe, respectively) is also consistent with a larger magnetic anisotropy for the nanochains. ${ }^{51,52}$

The observation of magnetic anisotropy indicates that fillers not only aggregate forming oriented needles but also tend to partially originate-in the same direction of the needles - an easy magnetic axis associated to the fillers. In general, this effect may arise from crystalline magnetic anisotropy (expressed by the magnetic anisotropy constant, K) or from shape anisotropy. The results presented here and the discussion at the end of the previous paragraph indicate that in the case of the $\mathrm{Ni}$ nanochains the main contribution to the magnetic anisotropy seems to come mostly from anisotropic shape morphology, that is from the shape of the fillers which is defined previously to cure. This is in accordance with the larger magnetic anisotropy observed for the case of composites with nanochains. The hypothesis favoring shape effects over crystalline magnetic anisotropy is reinforced by noting that the absolute value of the magnetic anisotropy constant, $\mathbf{K}_{\text {bulk }}$, for nickel is one order of magnitude lower than for many magnetic compounds (e.g. $\quad \mathbf{K}=5 \times 10^{4}$ $\mathrm{erg} / \mathrm{cm}^{3}$ for $\mathrm{Ni} ; \mathbf{K}=1 \times 10^{5} \mathrm{erg} / \mathrm{cm}^{3}$ for $\mathrm{Fe}_{3} \mathrm{O}_{4} ; \mathbf{K}=4 \times 10^{6}$ $\mathrm{erg} / \mathrm{cm}^{3}$ for $\left.\mathrm{CoFe}_{2} \mathrm{O}_{4}\right){ }^{47}$

\section{E. Elastic behavior of PDMS-Ni magnetorheological composites}

Stress-strain curves were recorded for both directions, that is, compressing in the directions parallel (//) and perpendicular $(\perp)$ to the needles. Different elastic tests were performed for magnetorheological composites with $2 \% \mathrm{Ni}$ based nanoparticles and nanochains such as: (a) compressing at constant speed $(100 \mu \mathrm{m} / \mathrm{s})$ up to reaching a maximum strain (typically 30\%) followed by a sudden release, (b) 
keeping the compression at the maximum strain for several minutes, (c) repeating compression-decompression cycles. In no case elastic hysteresis was observed (the decompression curve matches the compression one). During compression the equipment records the pressure, $\mathbf{P}$, as function of the strain, $\boldsymbol{\varepsilon}$ (see definition in Eqs. (2a) and (2b)). The qualitative aspects of these curves are similar to those obtained for PDMS composites when using $\mathrm{CoFe}_{2} \mathrm{O}_{4}$ and $\mathrm{Fe}_{3} \mathrm{O}_{4}$ as fillers, reported in previous works of our group. ${ }^{2-5,49}$

For a given sample the strain-stress curves were not modified after at least ten cycles. No relaxation of the material was observed when keeping fixed a $30 \%$ strain during $10 \mathrm{~min}$. The increase of pressure during compression was higher when compressing in the direction parallel than for compression perpendicular to the needles. That is, we have observed the induction of anisotropic effects without any hysteresis for all the prepared samples. These conclusions hold for composites made with nanoparticles and nanochains. Hence, these results indicate that the composites behave as anisotropic elastic materials with no elastic memory effects under the indicated conditions.

We propose here that the degree of elastic anisotropic effects can be taken into account by introducing the parameter $\boldsymbol{A}_{\text {elastic }}$, defined by

$$
A_{\text {elastic }} \equiv 100 \frac{\left(\mathbf{L n}\left(1-\varepsilon_{\perp}\right)-\mathbf{L n}\left(1-\varepsilon_{/ /}\right)\right)}{\mathbf{L n}\left(1-\varepsilon_{/ /}\right)},
$$

where the symbol $\varepsilon$ stands for the strain on the sample (in absolute values)

$$
\begin{gathered}
\varepsilon_{\perp} \equiv \frac{L_{i \perp}-L_{\perp}}{L_{i \perp}}, \\
\varepsilon_{/ /} \equiv \frac{L_{i / /}-L_{/ /}}{L_{i / /}},
\end{gathered}
$$

with $0 \leq \varepsilon_{/ /}, \varepsilon_{\perp} \leq 1$. The symbols // and $\perp$ refer to compressions in the direction parallel and perpendicular to the needles, respectively. $\boldsymbol{L}_{\boldsymbol{i}}$ represents the initial thickness previous to initiate the compression (note that $\boldsymbol{L}_{\mathbf{i}}$ may be not exactly the same for both directions) and $\boldsymbol{L}$ the actual sample's thickness when a pressure is applied. The parameter $\boldsymbol{A}_{\text {elastic }}$ is very convenient in order to compare the elastic anisotropy of the two magnetorheological elastomers, with nanoparticles vs. nanochains, since it is not based in any model assumption (like Young's or Poisson's laws) and is defined using the variables which are obtained experimentally (the strains).

The obtained values of $\boldsymbol{A}_{\text {elastic }}$ are independent of the applied pressure and considerably larger for the composites with nanochains than for those prepared with nanoparticles: $\boldsymbol{A}_{\text {elastic }}=21 \%$ and 3\% for PDMS-nanochains and PDMSnanoparticles composites, respectively $(2 \% \mathrm{w} / \mathrm{w}$ filler loading in both cases). Hence, as in the case of the magnetic anisotropy, the composites with nanochains render the largest elastic anisotropy. Figure 9 illustrates this difference in the anisotropic elastic behavior between composites using nanoparticles and nanochains by representing the absolute value of the numerator in Eq. (1), $\left|\operatorname{Ln}\left(1-\varepsilon_{\perp}\right)-\operatorname{Ln}\left(1-\varepsilon_{/ /}\right)\right|$,

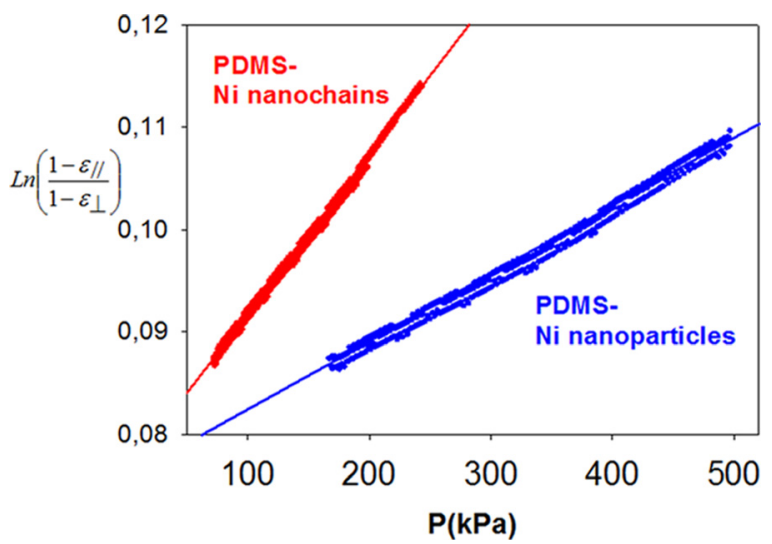

FIG. 9. $\operatorname{Ln}\left(\frac{1-\varepsilon_{/ /}}{1-\varepsilon_{1}}\right)$ versus the applied pressure P, for PDMS structured composites using Ni-based nanoparticles and nanochains. The strains $\varepsilon_{/ /}$and $\varepsilon_{\perp}$ are defined in Eqs. (2a) and (2b).

as function of the applied pressure $\mathbf{P}$ for both types of composites. The slopes of the straight lines shown in Figure 9 are expected to be proportional to the difference of the compression modules in both directions (neglecting lateral dilatation related to the Poisson's modulus), thus, the higher slope obtained for the case of composites with $\mathrm{Ni}$ nanochains reflects its higher elastic anisotropy. It is important to note that the comparison of the absolute values of the elastic parameters in magnetorheological structured composites can lead to erroneous conclusions, since they are influenced by details of sample preparation which are not easy to match perfectly: mass of the magnetic nanoparticles, intensity of the magnetic field during curing, relative amounts of base and cross-linker, time and temperature of curing, etc.

Stress-Strain curves were performed in the presence and absence of a magnetic field, by placing an anisotropic $2 \%$ $w / w \mathrm{Ni}$ (nanoparticles)-PDMS composite on the surface of a rare-earth disk-shaped magneto (axially magnetized) and by compressing the sample from above with the Texture Analysis device (described in Sec. II G). The diameters of the composite and magnet were $1 \mathrm{~cm}$ and $3.6 \mathrm{~cm}$, respectively; the thickness of the composite $3.5 \mathrm{~mm}$ approximately. Curves in the absence of the magneto were first recorded. Then the magnet was located on the device and the samples placed on the magneto. An elapsing time of 5 min was considered before running the stress-strain curves, allowing the material to relax. The magnetic field at the center of the magnet was measured with a Hall-probe, obtaining $160 \mathrm{mT}$ at the center and on its surface. It is worth to mention that the magnetic field is non-uniform and decays rapidly with the distance from its surface $(\mathrm{Z})$ with $\mathrm{Z}^{-4}$. Hence, the following results must be considered only as a demonstration of magnetorheological effects when the sample is exposed to a particular magnetic field.

Different tests were performed, recording the pressure, $\mathbf{P}$, on the sample as function of time, $\mathbf{t}$ during the test $(\mathbf{t}$ is proportional to the parallel strain, $\boldsymbol{\varepsilon}_{/ /}$, since the speed of compression is constant) (Figure 10). Tests were performed first in the absence and then in the presence of a magnet (the curves of the respective tests were then overlapped for a better visualization): 
(a) Samples were compressed from the top and parallel to the needles, at constant speed $(100 \mu \mathrm{m} / \mathrm{s})$ up to reaching a maximum strain (40\%) followed by a sudden release of compression, Figure 10(a). Three cycles of this type were consecutively performed in each case (presence and absence of the magneto). (b) The compression at the maximum strain was kept for $10 \mathrm{~s}$ and the compressing probe was suddenly released (Figure 10(b)). (c) Idem (b), but the probe is slowly released at the same speed used for compressing $(100 \mu \mathrm{m} / \mathrm{s}$, Figure $10(\mathrm{c}))$.

It is observed that the forces required for reaching a given strain are always lower for samples placed on the

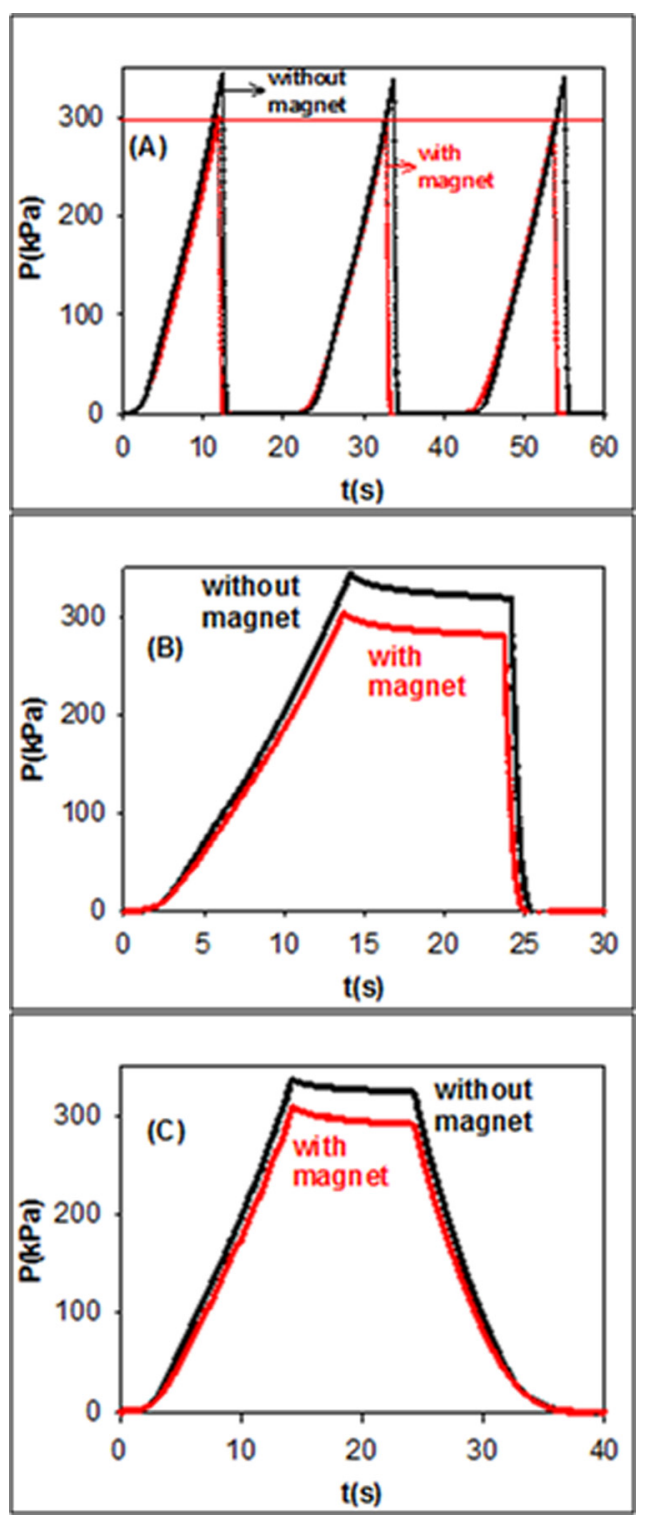

FIG. 10. Stress-strain curves in presence and absence of a magnet close to the sample. A $2 \% w / w \mathrm{Ni}$ (nanoparticles)-PDMS structured composite is placed on the top of a disk-shaped magnet. Composite diameter: $1 \mathrm{~cm}$. Magnet diameter: $3.6 \mathrm{~cm}(\mathrm{~B}=160 \mathrm{mT}$ at the center on the surface). $\mathrm{P}(\mathrm{kPa})$ represents the pressure exerted on the sample during compression in the direction parallel to the needles; $t(s)$ is the elapsed time (in seconds). (a) Samples are compressed at constant speed up to reaching a maximum strain (40\%) followed by a sudden release of compression. (b) Compression at the maximum strain was kept for $10 \mathrm{~s}$ and then the compressing probe was suddenly released. (c) Idem (b), but the probe is slowly released at the same speed used for compressing. Speed of compression and maximum strain: $100 \mu \mathrm{m} / \mathrm{s}$ and $40 \%$, respectively, in the three cases. magnet. Although these results must be considered as preliminary, the decrease of force/pressure required for reaching a given strain and the different features observed in Figures 10(a)-10(c) may be interpreted by considering the deformations of the initially more structured configuration of aligned needles induced by the spatial non-uniformity of the field, with an heterogeneous disruption of the order due to the strong dependence of the field with the distance along the composite. This heterogeneous disruption of the order may be responsible of the lower response force and the reduced thickness observed when samples are placed at the top of the magneto. These results were perfectly reproducible in several repetitions and clearly indicate an effect of the magnetic field on the elastic behavior of the material. However, a quantitative description of the magnetorheological effect requires a full systematic study under uniform magnetic fields which s outside the scope of the present work.

\section{CONCLUSIONS}

Nickel-based nanoparticles and nanochains were obtained by synthetic routes which do not require argon atmosphere or high temperatures. In the case of the particles, single-crystal, magnetic mono-domain, fcc nanoparticles with high chemical purity were obtained, verified by XRD, SAXS, TEM, SEM, EDS, and VSM. The nanoparticles can be well described as a polydisperse system of spherical hardspheres (distribution centered at about $10 \mathrm{~nm}$ diameter according to SAXS), displaying ferromagnetic behavior with $\mathbf{M}_{\mathbf{s}}=20 \mathrm{emu} / \mathrm{g}, \mathbf{M}_{\mathbf{r}}=5.4 \mathrm{emu} / \mathrm{g}$ and $\mathbf{H}_{\mathbf{c}}=180 \mathrm{Oe}$. The lower values of $\mathbf{M}_{\mathbf{s}}$ and $\mathbf{M}_{\mathbf{r}}$ in comparison with those for bulk are in agreement with the model clearly described in Ref. 46, where the effects of a non-magnetic layer surrounding the particles (due to spin-disorder at the surface) becomes relevant as the nanoparticle size decreases and the surface/volume ratio increases. The increase of $\mathbf{H}_{\mathbf{c}}$ in comparison with the bulk value is in agreement with a magnetic monodomain description of the nanoparticles, since in that case the coherent rotation of spins is the prevalent factor for magnetic reversal, which requires more energy than processes that appear in bulk samples (like domain wall displacement). $\mathbf{M}_{\mathbf{s}}$, $\mathbf{M}_{\mathbf{r}}$, and $\mathbf{H}_{\mathbf{c}}$ for the nanochains are qualitatively consistent with that model having values in between those for the bulk and the nanoparticles.

The anisotropy effects (both magnetic and elastic) in the magnetorheological PDMS-Ni composites are larger when using nanochains as fillers than for the nanoparticles case, as expected from its larger aspect ratio (although not completely obvious since the fillers aggregates forming the needles). In fact, shape anisotropy of the fillers appears as the main factor for the FMR anisotropy observed in the composites. Hence, the results show that the Ni nanochains appear as a very promising material for potential applications of magnetorheological composites, moreover, considering that the synthesis can be relatively easily performed under ambient conditions.

A remarkable characteristic for the technological application of magnetorheological materials is that even using very low amounts of Ni-based nanoparticles or nanochains it 
is yet possible to induce anisotropy effects. The elastic response is sensitive to the presence of a magnetic field. The use of loading factors as small as $2 \% \mathrm{w} / \mathrm{w}$ (rendering volume fractions of the needles lower than $1 \%$ ) still induces magnetic and elastic anisotropy on the magnetorheological composites, as demonstrated by VSM, FMR, and the stress-strain analysis.

\section{ACKNOWLEDGMENTS}

P.S.A., G.J., A.B., and R.M.N. are research members of the Argentinean Research Council (CONICET). M.M.R. is a doctoral fellow of CONICET. Financial support was received from the University of Buenos Aires (UBACyT 2012-2015 and 200201101 00098), Ministry of Science, Technology and Innovations (MINCYT-FONCYT and PICT 2011-0377) and CONICET (PIP 6382). The authors thank to the Center of Documental Production (CePro) and the Center of Advanced Microscopy (CMA), School of Sciences, University of Buenos Aires, for obtaining the presented pictures and SEM-TEM images. C.L.P.O. was supported by FAPESP and INCTFcx, Brazil.

${ }^{1}$ N. Kchit and G. Bossis, J. Phys. Condens. Matter 20, 204136 (2008).

${ }^{2}$ J. L. Mietta, M. M. Ruiz, P. S. Antonel, O. E. Perez, A. Butera, G. Jorge, and R. Martín Negri, Langmuir 28, 6985 (2012).

${ }^{3}$ P. S. Antonel, R. M. Negri, A. G. Leyva, and G. A. Jorge, Physica B: Condens. Matter 407, 3165 (2012).

${ }^{4}$ P. S. Antonel, G. Jorge, O. E. Perez, A. Butera, A. Gabriela Leyva, and R. Martín Negri, J. Appl. Phys. 110, 043920 (2011).

${ }^{5}$ M. M. Ruiz, P. S. Antonel, O. E. Perez, R. Martín Negri, and G. Jorge, J. Magn. Magn. Mater. 327, 11 (2013).

${ }^{6}$ J. Zeng, Y. Guo, Y. Li, J. Zhu, and J. Li, J. Appl. Phys. 113, 17 A919 (2013).

${ }^{7}$ L. Chen and S. Jerrams, J. Appl. Phys. 110, 013513 (2011).

${ }^{8}$ D. Lorenzo, D. Fragouli, G. Bertoni, C. Innocenti, G. C. Anyfantis, P. D. Cozzoli, and R. Cingolani, J. Appl. Phys. 112, 083927 (2012).

${ }^{9}$ H. Høyer, M. Knaapila, J. Kjelstrup-Hansen, and G. Helgesen, J. Appl. Phys. 112, 094324 (2012).

${ }^{10}$ F. Semeriyanov, A. I. Chervanyov, R. Jurk, K. Subramaniam, S. Konig, M. Roscher, A. Das, K. W. Stockelhuber, and G. Heinrich, J. Appl. Phys. 113, 103706 (2013).

${ }^{11}$ J. D. Macias, J. Ordonez-Miranda, and J. J. Alvarado-Gil, J. Appl. Phys. 112, 124910 (2012).

${ }^{12}$ G. Diguet, E. Beaugnon, and J. Y. Cavaille, J. Magn. Magn. Mater. 321, 396 (2009).

${ }^{13}$ K. Keshoju and L. Sun, J. Appl. Phys. 105, 023515 (2009).

${ }^{14}$ H. Denver, T. Heiman, E. Martin, A. Gupta, and D.-A. Borca-Tasciuc, J. Appl. Phys. 106, 64909 (2009).

${ }^{15}$ O. Thompson Mefford, M. R. J. Carroll, M. L. Vadala, J. D. Goff, R. Mejia-Ariza, M. Saunders, R. C. Woodward, T. G. St. Pierre, R. M. Davis, and J. S. Riffle, Chem. Mater. 20, 2184 (2008).

${ }^{16}$ V. G. Stepanov, S. S. Abramchuk, D. A. Grishin, L. V. Nikitin, E. Yu. Kramarenko, and A. R. Khokhlov, Polymer 48, 488 (2007).

${ }^{17}$ G. Filipcsei, I. Csetneki, A. Szilágyi, and M. Zrínyi, Adv. Polym. Sci. 206, 137 (2007).
${ }^{18}$ E. Coquelle, G. Bossis, D. Szabo, and F. Giulieri, J. Mater. Sci. 41, 5941 (2006).

${ }^{19}$ A. Lappas Zorko, E. Wortham, R. N. Das, E. P. Giannelis, P. Cevc, and D. Arc, Chem. Mater. 17, 1199 (2005).

${ }^{20} \mathrm{M}$. Farshad and A. Benine, Polym. Test. 23, 347 (2004).

${ }^{21}$ M. R. Jolly, J. D. Carlson, and B. C. Muñoz, Smart Mater. Struct. 5, 607 (1996).

${ }^{22}$ S. M. Amir, M. Gupta, S. Potdar, A. Gupta, and J. Stahn, J. Appl. Phys. 114, 024307 (2013).

${ }^{23}$ N. Biziere, M.-C. Clochard, P. Do Chung, J.-E. Wegrowe, and M. Viret, J. Appl. Phys. 113, 173910 (2013).

${ }^{24}$ J. Lindroos, D. P. Fenning, D. J. Backlund, E. Verlage, A. Gorgulla, S. K. Estreicher, H. Savin, and T. Buonassisi, J. Appl. Phys. 113, 204906 (2013).

${ }^{25}$ L. Rosenthal, H. Greve, V. Zaporojtchenko, T. Strunskus, F. Faupel, and M. Bonitz, J. Appl. Phys. 114, 044305 (2013).

${ }^{26}$ D. J. Sprouster, R. Giulian, L. L. Araujo, P. Kluth, B. Johannessen, N. Kirby, and M. C. Ridgway, J. Appl. Phys. 109, 113517 (2011).

${ }^{27}$ A. Lahiri and Z. Tadisina, Mater. Chem. Phys. 124, 41 (2010).

${ }^{28}$ A. D. Omrani, M. A. Bousnina, L. S. Smiri, M. Taibi, P. Leone, F. Schoenstein, and N. Jouini, Mater. Chem. Phys. 123, 821 (2010).

${ }^{29}$ D.-P. Wang, D.-B. Sun, H.-Y. Yu, Z.-G. Qiu, and H.-M. Meng, Mater. Chem. Phys. 113, 227 (2009).

${ }^{30}$ Y. Chen, D.-L. Peng, D. Lin, and X. Luo, Nanotechnology 18, 505703 (2007).

${ }^{31}$ J. W. Park, E. H. Chae, S. H. Kim, J. H. Lee, J. W. Kim, S. M. Yoonb, and J.-Y. Choi, Mater. Chem. Phys. 97, 371 (2006).

${ }^{32}$ K. H. Kim, H. C. Park, S. D. Lee, W. J. Hwa, S.-S. Hong, G.-D. Lee, and S. S. Park, Mater. Chem. Phys. 92, 234 (2005).

${ }^{33}$ Y. Hou, H. Kondoh, T. Ohta, and S. Gao, Appl. Surf. Sci. 241, 218 (2005).

${ }^{34}$ K. H. Kim, Y. B. Lee, E. Y. Choi, H. C. Park, and S. S. Park, Mater. Chem. Phys. 86, 420 (2004).

${ }^{35}$ Y. Duan and J. Li, Mater. Chem. Phys. 87, 452 (2004).

${ }^{36}$ S. W. Lin, S. C. Chang, R. S. Liu, S. F. Hu, and N. T. Jan, J. Magn. Magn. Mater. 282, 28 (2004).

${ }^{37}$ H. Amekura, N. Umeda, Y. Takeda, J. Lu, and N. Kishimoto, Appl. Phys. Lett. 85, 1015 (2004).

${ }^{38}$ D. S. Sidhaye, T. Bala, S. Srinath, H. Srikanth, P. Poddar, M. Sastry, and B. L. V. Prasad, J. Phys. Chem. C 113, 3426 (2009).

${ }^{39}$ M. Alagiri, C. Muthamizhchelvan, and S. Ponnusamy, Mater. Lett. 65, 1565 (2011).

${ }^{40}$ Y. D. Li, C. W. Li, H. R. Wang, L. Q. Li, and Y. T. Qian, Mater. Chem. Phys. 59, 88 (1999).

${ }^{41}$ O. Glatter and O. Kratky, Small Angle X-ray Scattering (Academic Press, London, 1982).

${ }^{42}$ D. J. Kinning and E. L. Thomas, Macromolecules 17, 1712 (1984).

${ }^{43}$ J. S. Pedersen, Adv. Colloid Interface Sci. 70, 171 (1997).

${ }^{44}$ R. M. Negri, S. D. Rodriguez, D. L. Bernik, F. V. Molina, and A. Pilosof, J. Appl. Phys. 107, 113703 (2010).

${ }^{45}$ Y. Wang, Q. Zhu, and H. Zhang, J. Mater. Chem. 12, 1212 (2006).

${ }^{46}$ H. M. Lu, W. T. Zheng, and Q. Jiang, J. Phys. D: Appl. Phys. 40, 320 (2007).

${ }^{47}$ V. Blaskov, V. Petkov, V. Rusanov, L. M. Martinez, B. Martinez, J. S. Muñoz, and M. Mikhov, J. Magn. Magn. Mater. 162, 331 (1996).

${ }^{48}$ D. L. Leslie-Pelecky and R. D. Rieke, Chem. Mater. 8, 1770 (1996).

${ }^{49}$ A. Butera, N. Álvarez, G. Jorge, M. M. Ruiz, J. L. Mietta, and R. M. Negri, Phys. Rev. B 86, 144424 (2012).

${ }^{50}$ A. Butera, Eur. Phys. J. B 52, 297 (2006).

${ }^{51}$ A. Butera, S. S. Kang, D. E. Nikles, and J. W. Harrell, Physica B 354, 108 (2004).

${ }^{52}$ J. M. Vargas, R. D. Zysler, and A. Butera. Appl. Surf. Sci. 254, 274 (2007).

${ }^{53}$ See supplementary material at http://dx.doi.org/10.1063/1.4839735 for details of the implemented SAXS model. 